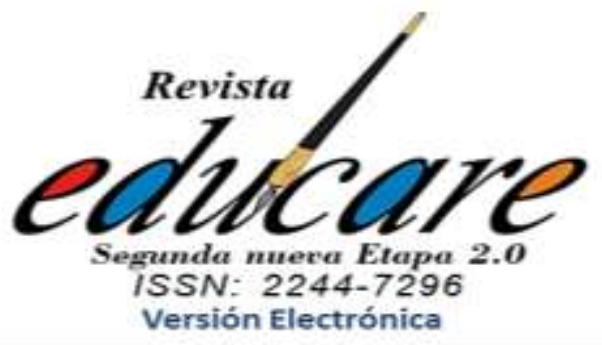

Volumen $25 \mathrm{~N}^{\mathrm{o}} 1$ Enero - Abril 2021

$(174-200)$

Julieht Alexandra Rodríguez Pérez *

ORCID: https://orcid.org/0000-0002-7762-7501

Ruth Stella Guerrero Godoy**

ORCID: https://orcid.org/0000-0002-3797-0145

Viviana Varon Arciniegas ****

ORCID: https://orcid.org/0000-0002-7762-7501

Benjamin Baron-Velandia*****

ORCID: https://orcid.org/0000-0002-4968-6336

Corporación Universitaria Minuto de Dios

- UNIMINUTO

(Colombia)

\title{
TRANSFORMACIONES DE LAS PRÁCTICAS PEDAGÓGICAS DE LOS PROFESIONALES NO LICENCIADOS
}

\author{
TRANSFORMATIONS OF THE PEDAGOGICAL \\ PRACTICES OF UNSODED PROFESSIONALS
}

* profesional en educación que cuenta con experiencia en la planeación, diseño, ejecución y evaluación de actividades lúdico-pedagógicas en primera infancia. Mi desempeño profesional en los distintos niveles de educación, no sólo me ha permitido llevar a cabo labores de docencia, sino también de investigación y proyección social, con lo que he desarrollado habilidades comunicativas, pedagógicas y administrativas requeridas, concernientes al ámbito educativo y la interacción social con distintas poblaciones en las que habitualmente me desenvuelvo.

** Psicóloga graduada y estudiante del último semestre de la maestría de Innovaciones Sociales en Educación de UNIMINUTO; tengo 4 años de experiencia laboral, relacionados con manejo de comunidades vulnerables, programa de primera infancia, capacitación y asesoría para la comunidad en comisaria de familia. ruth.guerrerog@uniminuto.edu.co

*** Estudiante de cuarto semestre de la Maestría en Innovaciones Sociales en educación, Licenciada en Pedagogía Infantil, con gran sentido de responsabilidad trabajo en equipo y proactividad, cuento con experiencia en docencia, coordinación e investigación; así como en la elaboración de propuestas educativas y proyectos. vvaronarcin@uniminuto.edu.co

**** Posdoctor en Epistemología. Doctor en Educación. Editor en Jefe de la revista científica internacional "Praxis Pedagógica". Director de Investigación (e) de la Corporación Universitaria Minuto de Dios UNIMINUTO Sede Bogotá Presencial. bbaron@uniminuto.edu

\section{Recibido: \\ 20-01-2021 \\ Aceptado: \\ 25-03-2021}



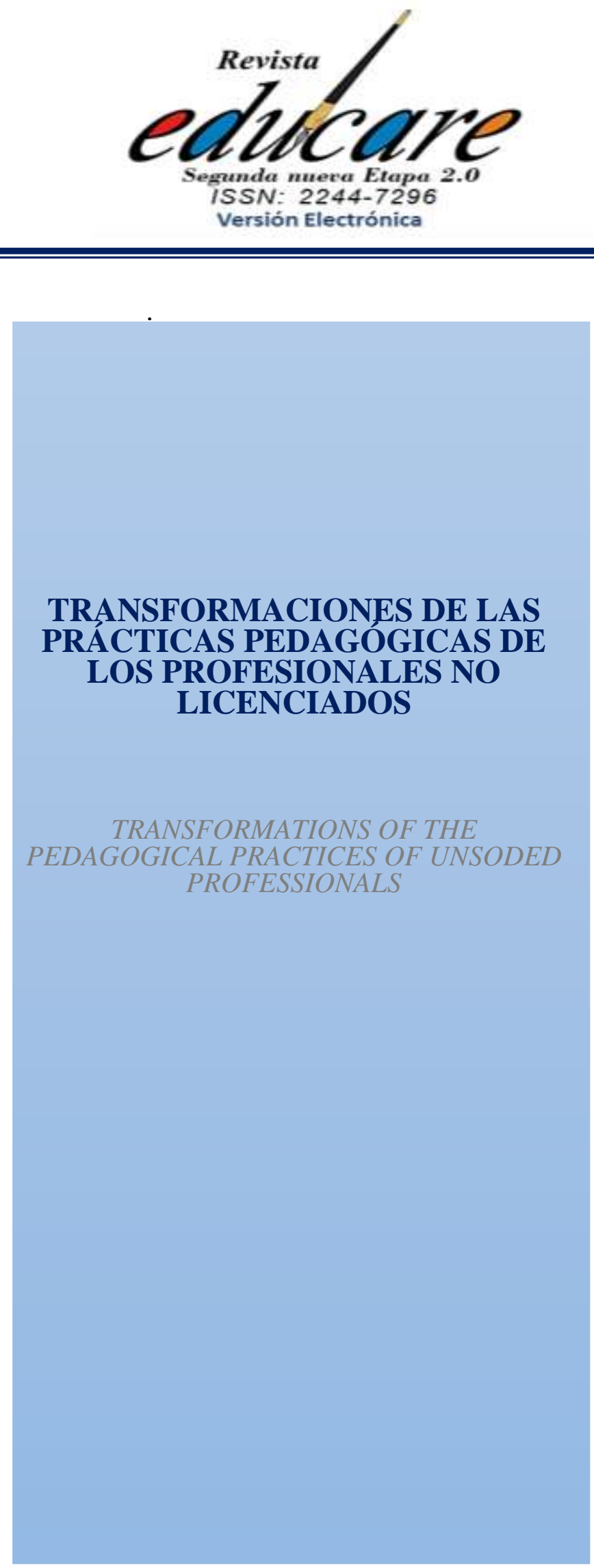

\title{
Resumen
}

El artículo tiene como propósito develar las transformaciones pedagógicas de los graduados del Programa de Pedagogía Para Profesionales No Licenciados (PPPNL) de la Escuela de Alta Docencia de la Facultad de Educación de UNIMINUTO durante el periodo 2018 y 2019. Se fundamentó en la investigación cualitativa- hermenéutica, la población sujeta de estudio estuvo conformada por 29 profesionales de la educación y la obtención de datos se realizó por medio del análisis de los Proyectos Pedagógicos de Aula, aplicación de encuesta y realización de entrevista semiestructurada. Los resultados mostraron que existen transformaciones significativas en las prácticas pedagógicas de los Profesionales No Licenciados y que el Programa de Pedagogía Para Profesionales No Licenciados es pertinente para la formación pedagógica de los mismos.

Palabras Clave: aprendizaje; formación pedagógica; práctica pedagógica; profesionales no licenciados.

\begin{abstract}
The purpose of the article is to reveal the pedagogical transformations of graduates of the Pedagogical Program for Unlicensed Professionals (PPPNL) of the High School of Education of UNIMINUTO during the period 2018 and 2019. It was based on qualitative- hermeneutical research, the population subject to study was made up of 29 professionals of the education and the obtaining of data was carried out by means of analysis of the pedagogical projects of Aula, application of survey and realization of semi structured interview. The results showed that there are significant transformations in the pedagogical practices of non-licensed professionals and that the pedagogy program for non-licensed professionals is relevant to the pedagogical training of those professionals.
\end{abstract}

Keywords: Learning; pedagogical training; pedagogical practice; unlicensed professionals. 


\section{Introducción}

Los Profesionales No Licenciados se han constituido en uno de los desafíos más grandes que enfrenta la educación colombiana, porque ante la posibilidad de acceso al sistema educativo, sin contar con una formación pedagógica adecuada que les permita adquirir las competencias pedagógicas: saber enseñar, organizar, desarrollar y dirigir situaciones y ambientes de aprendizaje; evaluar, proponer, desarrollar y sistematizar nuevas estrategias de aprendizaje y articular la práctica pedagógica con los nuevos contextos que les provee la cultura escolar (Ministerio de Educación Nacional, MEN, 2005).

En los últimos 15 años el sistema educativo colombiano ha experimentado un crecimiento de la incorporación de profesionales para ampliar la cobertura y mejorar las condiciones de calidad de la educación, en ese sentido mitigar las brechas socialmente existentes. En la actualidad más de 35 Instituciones de Educación Superior ofertan el programa de formación docente independiente del enfoque que caracterice el programa, en todos está presente la pedagogía que teoriza la educación en el territorio colombiano (MEN, 2021).

En relación con lo anterior, los últimos estudios demuestran que la formación de Profesionales No Licenciados se ha convertido en un compromiso ineludible de las diversas instituciones que componen el sistema educativo, posicionándolo como uno de los desafíos más importantes para los procesos de transformación social. En este orden de ideas, se evidencia la necesidad urgente de avanzar cada vez más con los desarrollos de programa que optimicen la adquisición de competencias pedagógicas para garantizar los procesos de enseñanza y aprendizaje, garantizando las competencias de los Profesionales No Licenciados, aseguramos un ambiente que desarrolle habilidades para aprender a lo largo de toda la vida.

En el sistema educativo colombiano hay una creciente de Profesionales No Licenciados que no cuenta con conocimientos pedagógicos y eso le imposibilita desarrollar de forma apropiada su labor docente.

El artículo da a conocer el estudio realizado con Profesionales No Licenciados que ejercen la labor docente y que cursaron el Programa de Pedagogía para Profesionales No 
Licenciados en la Corporación Universitaria Minuto de Dios - UNIMINUTO, de aquí en adelante UNIMINUTO. El objetivo de esta investigación fue develar las transformaciones pedagógicas de los graduados del Programa de Pedagogía para Profesionales No Licenciados (PPPNL) de la Escuela de Alta Docencia de la Facultad de Educación de UNIMINUTO durante el periodo 2018 y 2019; además, la investigación permitió conocer el impacto que tuvo el programa en el fortalecimiento de habilidades y destrezas de los Profesionales No Licenciados (PNL), de aquí en adelante (PNL) y en el desarrollo de su rol docente en la actualidad. El PPPNL de UNIMINUTO es uno de los programas más cursados de la Escuela de Alta Docencia y tiene como fin brindar una formación óptima a los PNL y contribuir con la construcción de la calidad educativa en el país. La Escuela de Alta Docencia de UNIMINUTO está comprometida con el propósito del sistema educativo del país, que es ofrecer una educación de calidad, y por esta razón, implementa diferentes estrategias que le permiten fortalecer sus procesos de enseñanza y aprendizaje. Fuente (consolidado de la oferta académica Escuela Alta Docencia, 2020).

\section{Argumentación teórica}

Rodríguez (2017) expone que el saber disciplinar, la pedagogía y la didáctica ofrecido en el programa contribuye a la mejora en la acción educativa del docente. En esta misma línea, se hace necesario mencionar que los Profesionales No Licenciados son definidos por García (2019) como: “Todo aquel profesional experto (tecnólogo, profesional, especialista, etc.) que, sin tener un título en licenciatura, pedagogía o ligado a la educación asume el rol de educador, es decir, asume prácticas profesionales propias de la educación” (pág. 36). En este sentido y teniendo en cuenta el (MEN, 2002) los autores de esta investigación exponen que los PNL son profesionales de la educación que ejercen la labor docente sin poseer un título de licenciados, pero que a través del decreto 1278 de 2002 se encuentran habilitados para el ejercicio de dicha labor en los niveles de básica secundaria y media en Colombia.

Por otro lado, es de mencionar que una de las razones de la inserción de los PNL al sistema educativo en el país fue contribuir con la mejora permanente de la calidad educativa; por esta razón, se considera oportuno que estos profesionales reciban formación pedagógica y se capaciten constantemente en su área de desempeño. En este sentido Pérez (2019) afirma "los docentes deben formarse didácticamente para ajustarse al modelo de enseñanza actual, 
permitiendo que sea el propio alumnado el que construya el conocimiento" (p. 3). De igual manera, los educadores están llamados a conocer el contexto sociocultural de los discentes para construir aprendizajes significativos Aktuna \& Oliveras (2016). En esta misma línea, es de resaltar que el PPPNL de UNIMINUTO promovió un conjunto de prácticas pedagógicas reflexivas en los PNL y demás docentes fundamentales en la construcción de la identidad de maestras/os, a saber, aprendizaje significativo, ecléctico y el constructivismo social con el propósito de mejorar la calidad educativa y formar seres humanos íntegros que la sociedad requiere en la actualidad (Fonseca, et al. 2019).

Para la OCDE (1995 (citado en Ropa, 2014, p.70) la educación de calidad es aquella que "asegura a todos los jóvenes la adquisición de los conocimientos, capacidades, destrezas y actitudes necesarias para equiparlos para la vida adulta". el sistema educativo se ha "concentrado en buscar la mejor manera de formar sujetos que aporten a una sociedad en materia de conocimientos y que se distingan a la hora de intervenir en la transformación de los espacios" (Varón, Martínez, León \& Barón, 2020, p. 490). Por lo cual, se entiende que la calidad educativa se logra cuando los procesos educativos satisfacen las necesidades y expectativas tanto del individuo como de la sociedad.

La calidad educativa es un concepto que agrupa varios enunciados o atributos que son dinámicos, es decir, que se encuentran en un constante cambio en pro de la mejora continua (Estrada, E., \& Mamani, H. 2020). De aquí, que asumir la calidad de la educación hace pensar en la responsabilidad de los docentes, por tanto, "se deben promover procesos para su mejoramiento profesional a través de programas de excelencia para su formación" (Rodríguez e Hinojo, 2018, p. 54).

Para dar cumplimiento al objetivo de investigación se hizo necesario ampliar el conocimiento sobre prácticas pedagógicas y aplicar diferentes estrategias para obtener la información. Las prácticas pedagógicas son la columna vertebral del ejercicio docente, es decir, el alma de la docencia, al respecto Zambrano (2006 Ibid) afirma "la pedagogía expresa unas formas de decir y al mismo tiempo unas prácticas de hacer” (p. 395).

En la actualidad, existen muchas definiciones sobre prácticas pedagógicas, las cuales en su mayoría de caso convergen entre sí, pero en este caso nos situamos en la comprensión de Zuluaga (1987) quien define que la práctica pedagógica “constituye un escenario histórico no solamente de la enseñanza sino también del maestro, la escuela, el método, el aprendizaje, 
la formación y de los estudiantes" p. 24, lo que nos permite afirmar que las prácticas pedagógicas son las diversas acciones que realiza el docente en pro de la formación integral a sus estudiantes, y estas reflejan la fundamentación teórico - práctica que posee el profesional con el tema o actividad trabajada, de igual manera Hammond (2006, 2012) reconoce la correlación entre la calidad de la formación y el desempeño de los profesores en contextos escolares.

En esta misma línea, Durán (2014) afirma “a diario, los docentes se enfrentan a múltiples funciones, exigencias sociales, multiplicidad de identidades, conflictos y políticas educativas, que los induce implícitamente a una continua reflexión sobre su labor y sobre su actuar"(p. 43); lo que nos permite discernir que los PNL y los demás profesionales de la educación, que ejercen la labor docente deben estar en constante formación, dado que esto es lo que permite que ellos brinden una orientación y formación óptima a sus estudiantes, de igual manera Figueroa (2018) afirma que "la reflexión en y sobre la práctica ha marcado los planes de formación en algunas instituciones imprimiendo un sello en la formación de profesores de carácter reflexivo y analítico”. (p. 113).

Adicionalmente, Zuluaga (1987) expone que el concepto de práctica pedagógica involucra una relación de tres instancias (maestro, saber y escuela) y que estas no son estáticas, debido a que se modifican de acuerdo con las necesidades de la sociedad del momento (Bracho Pérez, 2019). Desde este punto de vista, se entiende que las prácticas pedagógicas varían en el tiempo y en la escuela, dado a que estas se transforman y adaptan en relación con el modelo pedagógico institucional.

Las prácticas pedagógicas son el escenario donde el maestro dispone de elementos propios de su personalidad académica y personal, en este sentido Di Franco y Gentile, (2013) manifiesta que, en la formación docente, la práctica venía siendo concebida como una instancia final, como un momento, en el cual el profesional debería demostrar lo aprendido.

Los docentes del siglo XXI estamos llamados a construir nuevos caminos que permitan transformar la manera de pensar y de actuar para un mayor desarrollo de la educación y la acción de educar y educarnos. Escudero (2018). Las prácticas constituyen una oportunidad en la que profesores en formación comparten los conocimientos teóricos aprendidos hacia escenarios y contextos educativos. (Jarpa, Haas \& Collao, 2017). 
De igual manera, las prácticas pedagógicas se conciben como un proceso de autorreflexión, que se convierte en el espacio de conceptualización, investigación y experimentación didáctica, donde el profesional docente aborda saberes de manera articulada y desde diferentes disciplinas que enriquecen la comprensión del proceso educativo y de la función docente en el mismo; en este sentido Firdyiwek y Scida (2014) afirman que la reflexión pedagógica es un trasmisor de aprendizaje, capaz de proponer nuevas técnicas de enseñanza, así, el proceso reflexivo del docente debe ser una tarea sistemática en el desarrollo de su quehacer. (Ídem).

Por tanto, no es una falacia afirmar que la reflexión de las prácticas pedagógicas promueve el desarrollo de las competencias profesionales de los docentes y coinciden en un doble sentido: primero, como práctica de enseñanza propia del proceso formativo, segundo como la apropiación del oficio docente buscando cómo iniciarse en la profesión, perfeccionarse y actualizarse en su rol como formador y productor de nuevos conocimientos. Por esta razón la importancia de aplicar diferentes estrategias para conocer los contenidos temáticos del programa y la influencia que pueden generar estos contenidos sobre la función docente de los PNL.

Teniendo en cuenta los resultados obtenidos en esta investigación, se puede afirmar que la organización del pensum del PPPNL de UNIMINUTO, permite que los profesionales no licenciados adquieran una variedad de conocimientos que le fortalecen sus habilidades y mejoras en sus procesos de interaprendizaje (Torres, J., \& Flores Ferrer, K. M. 2018). Lo anterior, posibilita la retroalimentación positiva y negativa del programa para ajustar sus contenidos, metodologías, técnicas, por ejemplo, la implementación de una comunidad de aprendizaje donde los participantes interactúen libremente con otros profesionales realice diferentes actividades pedagógicas, den respuesta a problemáticas educativas, busquen respuestas a inquietudes, colaboren en la construcción de conocimientos, investiguen de manera conjunta, entre otras. Adicional a lo anterior, es necesario tener en cuenta tanto las voces de los PNL como las de los docentes participantes como una herramienta esencial para el fortalecimiento del programa y la construcción de una educación de calidad.

\section{Método y materiales}

El diseño metodológico utilizado en esta investigación fue el enfoque cualitativohermenéutico, que permite atender, resolver y situar la realidad social y educativa del 
individuo, es decir, comprender todos los contextos de interacción de la persona. Al respecto Bonilla \&Rodríguez (2005) afirman que "la característica de la investigación cualitativa es un proceso de entradas múltiples que se retroalimentan con la experiencia y el conocimiento que se va adquiriendo de la situación" (p.121). La investigación cualitativa permite captar la realidad social a través de los ojos de la gente que está siendo estudiada, es decir, a partir de la percepción que tiene el sujeto de su propio contexto; actualmente, el enfoque cualitativo ha ido ganando credibilidad, al momento de hacer investigación Borjas (2020).

En esta misma línea, es esencial destacar el papel de la hermenéutica en la investigación, porque permite reconocer y comprender las realidades educativas, sociales, culturales, políticas y económicas de los contextos y actores indagados. En este sentido, Grodin (2002) define a la hermenéutica como "la pretensión de explicar las relaciones existentes entre un hecho y el contexto en el que acontece" (p.4). De igual manera expone que "la hermenéutica, por ejemplo, está asociada al arte de la interpretación de los textos para comprender su sentido" (citado por López, 2013, p.9).

\section{Población y muestra}

Para Arias (2006) la población es un conjunto finito o infinito de elementos con características comunes para los cuales serán extensivas las conclusiones de la investigación esta queda delimitada por el problema y los objetivos del estudio (citado por Artigas \& Robles, 2010). De esta forma, la población sujeta a estudio estuvo conformada por 127 participantes, entre los cuales se encuentran 97 PNL y 30 docentes que direccionan los diferentes seminarios del PPPNL durante los años 2018 y 2019, de la Escuela de Alta Docencia - EAD de la Facultad de Educación de UNIMINUTO.

En relación con la muestra, para Hernández (2014) esta se define como un subconjunto de elementos que pertenecen a ese conjunto definido en sus características al que se le llama población. Para la investigación, se optó por una muestra no probabilística por conveniencia, es decir, que no se rige por las reglas matemáticas de la probabilidad, sino que permite métodos de observación que ayude a evaluar los criterios que recogen los intereses o propósitos de la misma. Lo anterior, garantizó la selección de la muestra que fue de 29 participantes (profesionales de la educación), de los cuales 17 son PNL y 12 docentes que dictan los seminarios. Es fundamental comprender que la muestra se compone de dos grupos, por un lado, los estudiantes (profesionales no licenciados) y por el otro, los docentes 
que direccionan los seminarios del Programa de Pedagogía para Profesionales No Licenciados (PPPNL).

Las herramientas de recolección de datos utilizadas en esta investigación permitieron seleccionar, analizar, comprender y gestionar la información e integrar las tecnologías para un acercamiento con la población de estudio. (Ramírez, González \& López, 2020).

El primer grupo de selección fue los PNL, el cual se inicia con una carta de invitación electrónica a los 97 PNL graduados del PPPNL, pertenecientes a las cohortes 57, 58, 59 y 61; pero solo se logró contactar a 60 PNL; por esta razón nuestra población sujeta de estudio fue de 60 PNL. Posteriormente, se contactaron vía telefónica a esta población, dado que manifestaron por vía electrónica su interés de participar en la investigación. Luego, se evaluó quienes cumplen con los siguientes criterios de selección, dando como resultado una muestra de 17 PNL que cumplieron con los siguientes criterios de selección:

1. Ser graduados del PPPNL entre los años 2018-2019.

2. Estar de acuerdo con el objetivo de investigación.

3. Ejercer la docencia en la actualidad.

4. Tener Presente el Proyecto Pedagógico de Aula que se implementó.

5. Compartir las transformaciones de sus prácticas pedagógicas.

6. Querer divulgar las experiencias vividas en el programa.

\section{Gráfico 1}

Graduados del Programa de Pedagogía Para Profesionales No Licenciados

Población invitada

Población interesada

Muestra criterios de selección

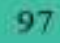

60

El segundo grupo de selección estuvo conformado por 30 docentes que pertenecen al PPPNL de UNIMINUTO, en donde se inicia con una carta de invitación para participar en la investigación, donde solo 12 docentes manifestaron interés como se puede observar en la siguiente gráfica: 


\section{Gráfico 2}

Docentes del Programa de Pedagogía Para Profesionales No Licenciados

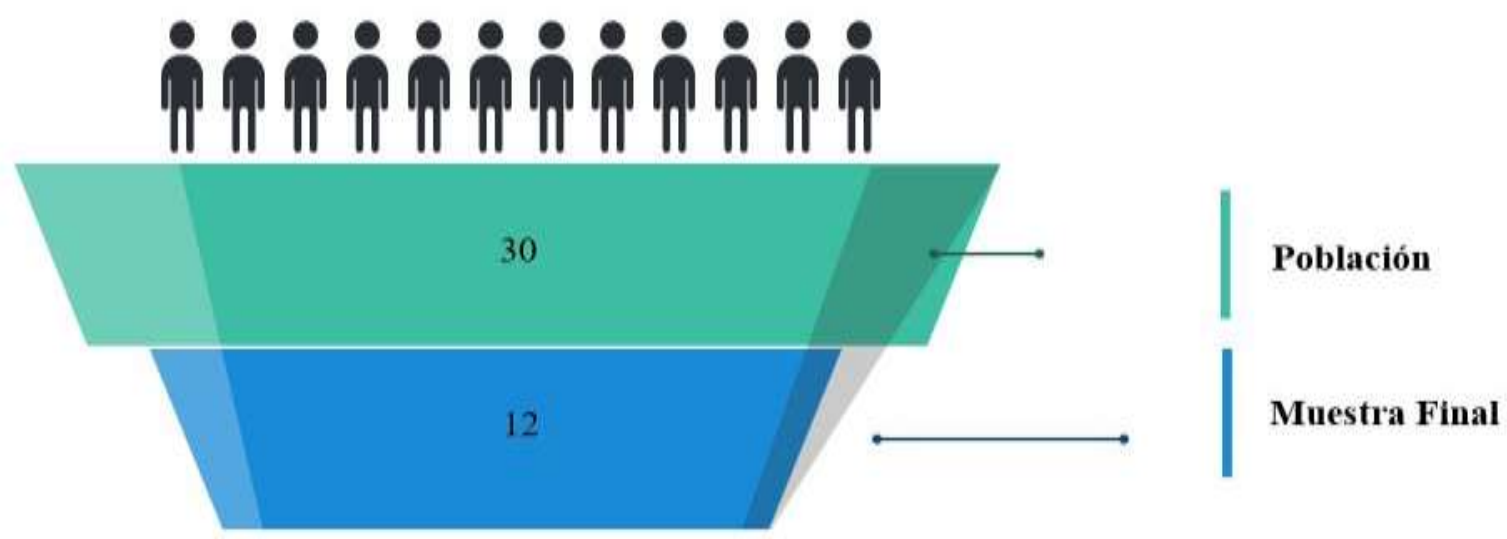

El total de la muestra sujeto de estudio de esta investigación fueron 29 profesionales de la educación.

\section{Técnicas e instrumentos de recolección de datos}

Las técnicas e instrumentos utilizados para recolectar la información de esta investigación fueron tres (revisión documental, encuesta y entrevista semiestructurada):

- Revisión Documental: Con relación al análisis de documentos como instrumentos de recolección de datos Quintana (2006) afirma que el análisis documental constituye el punto de entrada a la investigación y en muchas ocasiones, es el origen del tema o problema de investigación. Por consiguiente, la revisión de documentos en este caso fue el estudio minucioso de los proyectos pedagógicos de aula de los 17 graduados; para ello, se realizó un instrumento llamado: Fuente de revisión de datos PPA, en donde se sistematizó la información esencial que permitió conocer: el planear, ejecutar y actuar de los PNL.

Posteriormente, en los PPA se analiza: el modelo pedagógico que se utilizó durante su implementación, objetivos propuestos y alcanzados, resultados obtenidos, conclusiones y prospectivas; con esta revisión se identificó el impacto que tuvieron los proyectos en la sociedad, qué estrategias aportaron y el nivel de significatividad de la experiencia en la vida de los participantes. 
- Encuesta: De acuerdo con Buendía (1998) la encuesta es el "método de investigación capaz de dar respuestas a problemas tanto en términos descriptivos como de relación de variables, tras la recogida de información sistemática, según un diseño previamente establecido que asegure el rigor de la información obtenida" (p.12). La investigación ratifica que a partir de este instrumento se recolectaron datos importantes que contribuyeron con la consecución del objetivo trazado. Por esta razón, se optó por la aplicación de una encuesta online, la que facilitó la gestión de la información por cuanto brindó autonomía a los participantes para responderla. Esta, estuvo conformada por 16 preguntas mixtas, que buscaban develar información sobre: su formación, labor docente, experiencia en el PPPNL, e implementación del PPA.

- Entrevista Semiestructurada: Para Ryen (2013) \& Grinnell y Unrau (2011) definen que las entrevistas semiestructuradas "se basan en una guía de asuntos o preguntas y el entrevistador tiene la libertad de introducir preguntas adicionales para precisar conceptos u obtener más información" (citado por Hernández, 2014, p. 436). Como se señala, la entrevista semiestructurada permitió plantear interrogantes que tuvieran como propósito la comprensión del problema, sobre el instrumento en cuanto artefacto, ese desligue de la secuencia establecida que plantea un instrumento aporta valor a la configuración de reestructurar preguntas y rearmar contextos en la indagación con mayor profundidad.

Se aplicaron dos entrevistas semiestructuradas a la muestra. La primera, dirigida a los graduados del PPPNL, la cual tuvo como objetivo conocer sus prácticas pedagógicas subyacentes en los períodos 2018 y 2019; este instrumento, compuesto por 22 preguntas de carácter mixta, los entrevistados fueron libres de brindar y compartir información de manera espontánea y confiada. La segunda entrevista se aplicó a los docentes, esta tenía como objetivo compartir experiencias de transformación de los educadores que dirigen algunos seminarios, para aportar desde su lugar de observación, las mejoras en el desarrollo del programa; este instrumento, conformado por 28 preguntas de carácter mixto, en donde los participantes compartieron información valiosa para el cumplimento del objetivo propuesto.

Por otra parte, es importante resaltar que estas entrevistas, se aplicaron online, con el fin de proponer un escenario de mayor libertad para responder, proponer un espacio de confianza, analizar sus puntos de vista, la comprensión acerca del tema, reconocer sus transformaciones durante el PPPNL y analizar el tono de voz y sus expresiones. 
La validación de los instrumentos se realizó a través de la siguiente dinámica. Elaboración de los cuestionarios, aplicación de una prueba piloto, refinamientos de las preguntas, verificación de la consistencia de las respuestas contra las preguntas y validación por expertos externos.

\section{Resultados}

La formación pedagógica que ofrece el PPPNL de UNIMINUTO, fomentó en los PNL sus transformaciones en las prácticas pedagógicas, evidenciándose aspectos como una mejor planeación de sus clases y un mayor acercamiento con los discentes durante el proceso de formación.

En el siguiente gráfico (\# 3) se observa el ascenso en el comportamiento de las transformaciones de las prácticas pedagógicas que se obtuvieron durante el proceso investigativo.

\section{Gráfico 3}

Transformaciones de las prácticas Pedagógicas

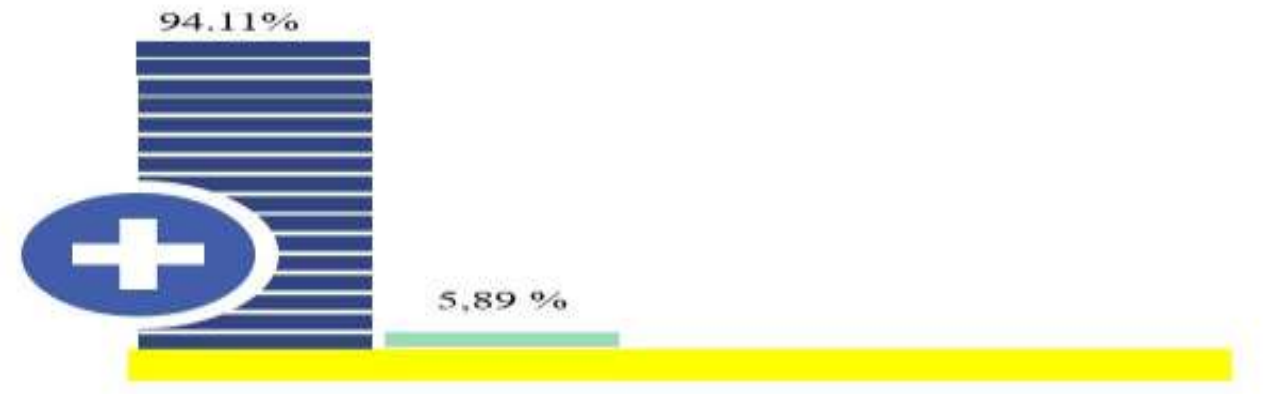

De los resultados obtenidos en la encuesta de percepción a los 17 participantes gráfico (\# 3) se evidenció que un total de 16 PNL que equivalen al 94.11\%, manifestaron haber transformado sus prácticas pedagógicas durante el proceso de desarrollo del programa, por el contrario, 1 de los PNL que equivale al 5,89 \% no percibió transformaciones en estas.

Como se puede evidenciar en el gráfico (\# 4) los PNL resaltan que los seminarios que más le aportaron en su formación fueron: modelos pedagógicos, ambientes de aprendizajes TIC, psicología educativa, innovación pedagógica y política educativa, lo que les brindó una formación dialéctica entre teoría y práctica oportuna para que estos profesionales reflexionaran e innovaran sus prácticas pedagógicas. 


\section{Gráfico 4}

\section{Seminarios más significativos}

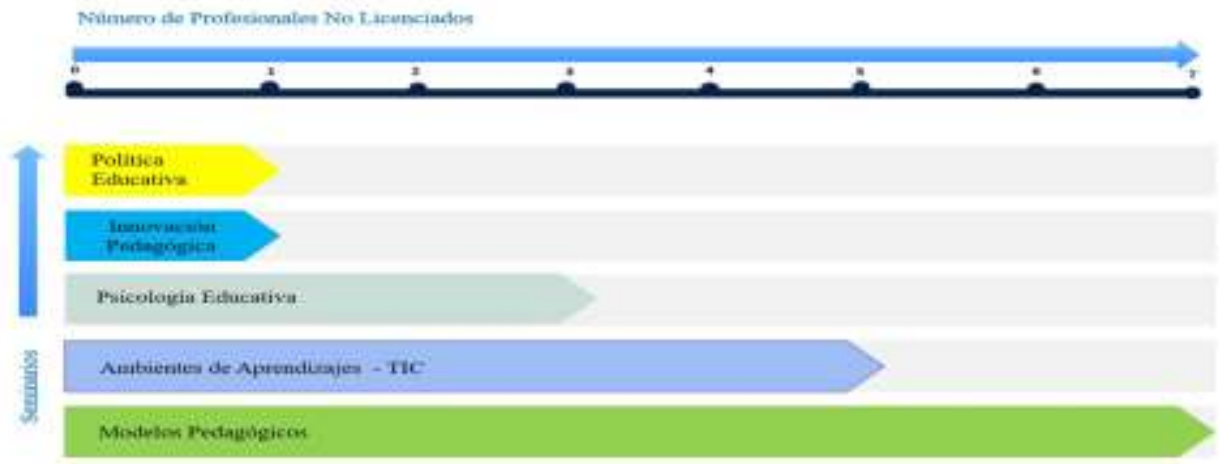

La experiencia adquirida en los seminarios vistos durante el PPPNL les permitió que implementaran nuevas acciones en su quehacer docente, con el propósito de contribuir significativamente en el crecimiento integral de sus discentes.

El seminario modelos pedagógicos, porque les ayudó a establecer con qué modelo se sienten más identificados y a definir con cuál trabajar dependiendo del contexto; en cuanto al seminario de Ambientes de Aprendizaje y TIC les ayudó a conocer e implementar gran variedad de herramientas tecnológicas y crear diseños apropiados para la educación; en el seminario de psicología educativa, porque tuvo la oportunidad de reflexionar sobre el papel que tiene el maestro y la responsabilidad de cada uno como educador; respecto al seminario de innovación pedagógica les brindó la posibilidad de tener un proceso de definición, construcción y participación de estrategias pedagógicas que aportaran significativamente a la escuela del siglo XXI, Como colofón, el seminario de política educativa, aportó en la comprensión de interrogantes tales como ¿Cómo está diseñada la educación?, ¿Cuál fue su inicio?, ¿Por qué y para qué existe?, ¿Qué cosas han cambiado y que no? entre otros.

Existe una correlación entre el nivel de aportación del seminario y el nivel de apropiación del conocimiento por parte de los PNL, que se evidencia en compromiso con el desarrollo de los seminarios.

Como se puede evidenciar en el gráfico (\# 5) los PNL manifiestan que la forma de evaluar los procesos de enseñanza-aprendizaje fue transformada de manera significativa, donde implementaron en su proceso evaluativo, elementos de orden cualitativo y cuantitativo 
como consecuencia de lineamientos que devienen de los estándares educativos institucionales.

\section{Gráfico 5}

Evaluación

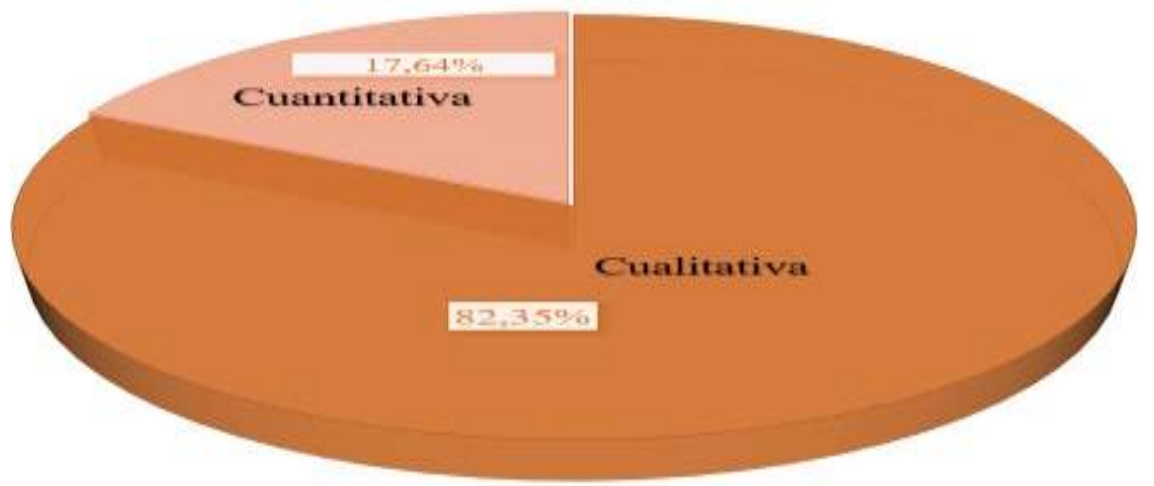

Se evidencia que 14 de los PNL equivalente al 82,35\%, manifestaron que utilizan en su proceso de evaluación, la evaluación cualitativa en donde se evalúan todos los aspectos (autoevaluación, heteroevaluación y coevaluación); por el contrario, 3 de los PNL equivalente al 17,64 \%, manifestaron que utilizan la evaluación cuantitativa en donde solo se tiene en cuenta las buenas respuestas de los estudiantes sin tener en cuenta sus cualidades y fortalezas.

Como se observa en el gráfico (\# 6) los PNL dan a conocer que la implementación del PPA les permitió tener transformaciones significativas en su rol como docente, las cuales fueron: tener una autorreflexión sobre todo su proceso formativo, evaluar y fortalecer su labor docente, sentirse orgulloso sobre su ejercicio como educador, estar siempre a la vanguardia de nuevos conocimientos e implementación de diferentes estrategias lúdico pedagógicas de nuevos procesos de formación fortaleciendo el trabajo en equipo permitiendo una construcción colectiva de conocimientos, tener autonomía de integrar nuevas prácticas en su quehacer, ser innovador en cuanto a los procesos de enseñanza-aprendizaje, integrar la evaluación cualitativa en su práctica como docente.

\section{Gráfico 6}

Transformaciones a partir de los Proyectos Pedagógicos de Aula 


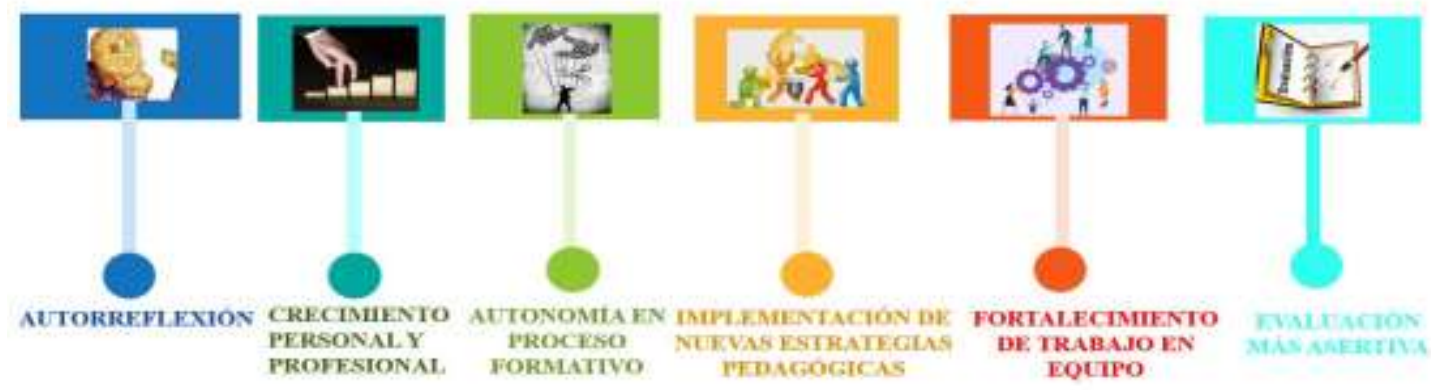

Los PPA proporcionan a los PNL un sin fin de herramientas que aportan significativamente a su formación como docente, respondiendo a la realidad social, cultural y económica del contexto educativo, lo que contribuye en sus prácticas pedagógicas dentro del aula de clase.

\section{Discusiones}

En este apartado se develan las 4 transformaciones más relevantes que tuvieron los Profesionales No Licenciados (PNL). Por ello, se hizo necesario analizar minuciosamente la información que compartieron los entrevistados Gauchi (2017).

Como primera medida, teniendo en cuenta la pregunta que interpelaba por la mejora del desempeño ¿Cree que ha cambiado la forma de orientar sus clases, a partir de su formación en el Programa Para Profesionales No Licenciados?, en este sentido Núñez (2002) citado por Tolozano, Lara, \& Illescas (2015, p. 116) afirma que "la formación pedagógica es la necesidad de hacer realidad una nueva manera de concebir y realizar la capacitación del docente en servicio, entendida esta como inherente al desarrollo de su práctica docente". En concordancia con lo anterior los docentes Arabugo, et al. (2020) manifiestan que "sí" ha cambiado la forma de orientar sus clases, puesto que consideran que la estructura del programa les permitió reflexionar sobre sus prácticas pedagógicas y les brindó herramientas esenciales para planear mejor sus clases, mejorar sus métodos de enseñanza, incluyendo la didáctica y las TIC, además, exponen que la formación pedagógica que recibieron en el programa, sin duda alguna permite que el maestro se transforme y pase por un proceso de aprendizaje no solo teórico sino práctico (Barón, 2017).

De acuerdo con la información anterior, se determina que la formación pedagógica recibida durante el programa de Pedagogía para Profesionales No Licenciados en UNIMINUTO brindó herramientas para enfocar su rol docente en los entornos educativos, 
reconociendo a ésta como la médula dorsal de los procesos de transformación individual y colectiva. De esta forma, se puede evidenciar que el programa generó en ellos la necesidad de entender y apropiarse todo lo que concierne a ser maestro en nuestro país (Pérez \& Ochoa. 2018). En esta misma línea, es necesario resaltar que los entrevistados reconocen que los contenidos temáticos del programa les permitió reflexionar sobre sus prácticas pedagógicas y ajustarlas o transformarlas teniendo como referentes las necesidades de sus estudiantes y el diseño pedagógico que fundamenta la institución educativa,

Adicionalmente, manifiestan que la reflexión les permite, una mirada mucho más comprensiva, es decir, dar cuenta de los errores cometidos, generar horizontes de posibilidades que los mitiguen, garantizar la corrección, pero más importante la prevención, un maestro que se relacione con seres humanos en procesos interculturales y debe ser sensible para vincularse con sus contextos lo que posibilitará impactar y transformar las vidas, el maestro se constituye en un referente a seguir no solo de sus estudiantes, sino también de la comunidad en la que está inmerso, el papel de profesor es multidimensional, vuelve su aula de clase en un escenario en el que el estudiante siente ánimo y esté dispuesto a recibir los procesos aprendientes(Varela-Ordorica \& Valenzuela-González, 2020). Si bien el rol del docente es primordial dentro del aula, ya que se constituye como una figura líder, el cual genera espacios para el trabajo colaborativo, así como lo manifestó Muñoz (2020).

El docente constantemente se forma para conocer y estar siempre a la vanguardia de los nuevos conceptos de la educación, se reconoce que los profesionales de la educación coconstruyeron conocimientos en su estructura de preparación de sus clases, pero ella va más allá, reconociendo que lo que desea realizar en sus clases es promover un aprendizaje significativo con sus estudiantes, en este sentido maestras y maestros, por nuestra proximidad a las nuevas generaciones, estamos llamados a comprender los paradigmas en los que estamos formados, para deconstruirlos e impactar la vida de quienes acompañamos en los procesos de aprendizajes, trascendiendo la comprensión cultural dualista de aprenderenseñar, en términos bancarios como lo plantearía Freire (citado por Brugaletta, 2017), e instalar una cultura de los interaprendizajes, en el que es fundamental el reconocimiento de quienes estamos en los procesos aprendientes Assmann (2003). Citado por Ferrada (2005) ver gráfico\# 3. 
En segunda instancia teniendo en cuenta la pregunta que interpelaba sobre ¿Cuál fue el seminario que más le aportó en su proceso de formación docente? los entrevistados León, Pulido, Padilla, Buitrago, Caldas, Rozo, Vanegas, Jara, Arabugo y Fernández (2020) exponen de manera general que fueron varios los seminarios que aportan en su proceso de formación, tales como "modelos pedagógicos, ambientes de aprendizajes y TIC, y psicología educativa", afirmando que estos seminarios fueron de "mayor incidencia para la transformación de sus prácticas pedagógicas", en concordancia con los autores anteriores Zuluaga(citado por Tamayo, 2004, p.30) define que la formación pedagógica “es el desarrollo de competencias para la conceptualización, aplicación y experimentación de los conocimientos, métodos y estrategias para la enseñanza de los saberes específicos en contextos institucionales”.

De acuerdo con lo anterior, se evidenció que los entrevistados coinciden en un primer momento que el seminario que más les aportó en su formación fue el de "modelo pedagógico", resaltando que este les permitió conocer los diferentes lineamientos que se debe tener en cuenta para la planeación y desarrollo de las actividades educativas y más para brindar una formación óptima a los discentes teniendo en cuenta sus particularidades tanto como cognoscitivas, afectivas y comunicativas (Pineda \& Orozco, 2016).

De igual manera, dan a entender que el seminario ambiente de aprendizajes y TIC, contribuyeron en las transformaciones de sus prácticas pedagógicas, debido a que el enfoque praxeológico del programa les permitió una constante reflexión del actuar en los diversos espacios educativos y fortalecer sus habilidades y destrezas, además, de la posibilidad de incorporar herramientas pedagógicas como la didáctica, las TIC en su rol docente (Juliao \& Barón-Velandia, 2013).

En consecuencia, con lo anterior Paredes y Velázquez (2020) consideran que la didáctica debe ajustarse a los procedimientos que produzcan aprendizajes significativos, y a la construcción de su propio aprendizaje, esto con el fin de crear espacios más didácticos en un ambiente confiable.

En esta misma línea se aseveró que la formación pedagógica es fundamental para un adecuado desarrollo de la labor docente, porque permite el crecimiento personal y profesional del formador o formador en formación, debido a que contribuye con el fortalecimiento de sus habilidades, destrezas y en la construcción de su identidad docente (Alzate-Ortizy CastañedaPatiño, 2020). Además, es de resaltar la importancia de esta formación para generar una 
reflexión del docente respecto a su quehacer en la escuela e introducir innovaciones necesarias en las prácticas pedagógicas y demás actividades académicas (Caldeiro-Pedreira y Castro-Zubizarreta, 2019).

De otra parte, el programa ofreció ambientes idóneos que facilitaron la formación pedagógica, didáctica y el desarrollo de competencias pedagógicas en los PNL, además, este ofrece una mejora en la docencia capacitando el perfil del docente con un listado de cualidades idóneas, inalcanzables ideales para la mejora educativa. Olmos, Sánchez y Correa (2016).

Asimismo, fue fundamental resaltar la formación pedagógica que se le brinda a los PNL en el PPPNL de UNIMINUTO contribuyó al fortalecimiento de sus conocimientos en pedagogía, métodos y estrategias de enseñanza-aprendizaje, procesos metacognitivos de la labor docente, reconozcan la importancia que tiene la formación y capacitación pedagógica en su crecimiento personal y profesional, además, estos docentes reconocen que para poder brindar una educación de calidad a los estudiantes y cumplir adecuadamente con las demás actividades educativas, es necesario recibir esta formación y continuar capacitándose en el área de formación. Ver gráfico (\# 4).

En tercera instancia, se hace necesario describir los hallazgos que surgieron a raíz de la pregunta que interpelaba por ¿Qué cambios ha evidenciado en las prácticas de evaluación del aprendizaje en sus estudiantes?, a lo que los entrevistados, León E, León M, Romero, Ramírez, Padilla, Buitrago, Flórez, Caldas, Rozo, Vanegas, Jara, Arabugo, González, \& Fernández (2020) exponen que los cambios realizados en el proceso de evaluación principalmente fueron la incorporación de "evaluación cualitativa", "la heteroevaluación y autoevaluación como criterios que procuran resultados más concretos sobre el proceso y logros alcanzados, además, la incorporación de la "expresión y creatividad” para realizar una evaluación más objetiva, y una "retroalimentación en el proceso de aprendizaje". En concordancia con los autores Zaccagnini (2008) define las prácticas pedagógicas como:

aquellas productoras de sujetos a partir de otros sujetos, es decir, se trata de una mediación, el rol de un sujeto mediador (sujeto pedagógico), que se relaciona con otro sujeto (educando) de esta relación surgen situaciones educativas complejas las que encuadran y precisan una pedagogía (citado por Morillo \& Quijano, 2016, p 323). 
El sujeto pedagógico se entiende como un ser histórico definido por la institución escolar, capaz de acercarse a esa complejidad de un universo sociocultural con una perspectiva más amplia que lo define con un sin número de articulaciones posibles entre educador, educando, saberes y la configuración de los espacios educativos que legitiman su propia pedagogía. Los entrevistados manifiestan que han cambiado sus prácticas pedagógicas con respecto a la evaluación, ya no se enfocan en la evaluación cuantitativa si no en la cualitativa, donde reconocen una multiplicidad de dimensiones de la persona tales como, el esfuerzo y los aprendizajes adquiridos por los estudiantes, actitud, compromiso, dedicación, aspiraciones e intereses (León, Risco, y Alarcón. 2018).

Además, incorporan la participación y la auto- evaluación en la complejidad de la evaluación, son más conscientes de que el aprendizaje no se reduce a los números, sino en la persona, le dan la importancia a la reflexión que hace el estudiante sobre sus aprendizajes en los procesos. En este sentido, la comprensión de la evaluación para los profesionales no licenciados dio un giro de 180 grados. Los procesos de autonomización en términos de Bourdieu (2012), se hace más dinámica y reflexiva. El estudiante reconoce el proceso como mucho más complejo, entendiéndolo como una oportunidad para reconocer sus prácticas en todo el proceso educativo, además de tener la retroalimentación del maestro, tiene un nuevo componente de observación crítica como es la de sus pares (Ayala, 2017).

Se evidenció que los entrevistados les asignan un papel más protagónico a sus estudiantes en el proceso de evaluación. Son esas buenas prácticas las que generan condiciones de posibilidad de cambio de la manera de evaluar en el entorno educativo, se reconoce que la evaluación cuantitativa es necesaria para cumplir con las notas administrativas que pide la institución, pero la comprensión de los aportes cualitativos es un reflejo que se puede representar en términos cuantitativos, Baron, Guerrero, Rodríguez, Varón (2020).

La construcción de los habitus en el campo de desarrollo de los aprendizajes, sacad el contexto de la competencia entendida como los que ganan y pierden, por la comprensión colaborativa de los procesos aprendientes (Zurita Aguilera, 2020). Anteriormente, el maestro tenía una comprensión sumativa del aprendizaje, la que estaba representada por "sumar las respuestas que tuvo bien el estudiante y eso daba la nota final", ahora con la incorporación de los nuevos aprendizajes, el profesional no licenciado, entiende que el sujeto, (estudiante) 
se le evalúa en todo su contexto, analizar el estilo, el ritmo, y la velocidad de su aprendizaje en el proceso educativo, brindarle oportunidades de reflexión al estudiante sobre sus aprendizajes y que él mismo reconozca el nivel de apropiación del conocimiento. Ver gráfico (\# 5).

Para terminar este análisis, se hace necesario describir los hallazgos de la pregunta que indagaba por ¿Cuáles fueron las transformaciones en los procesos de enseñanza y aprendizaje a partir de la implementación del proyecto pedagógico de aula? a lo que los entrevistados, plantearon que la implementación del proyecto permitió una "reflexión constante" sobre su nuevo ejercicio como docente, de igual manera, manifiestan "yo cambie la forma de tratar y ver a los estudiantes", "me permitió transformarme como persona y profesional de la educación, donde aprendí a reconocer y valorar las emociones y sentimientos de los estudiantes", "modificación de paradigmas", además, estos profesionales manifestaron que reconocen más procesos de "autonomía" a los discentes en su proceso formativo, la incorporación de "estrategias pedagógicas" en los procesos de interaprendizaje, que involucran su contexto e "incentivan a los estudiantes a trabajar en equipo y a realizar investigaciones. En concordancia con lo anterior Zaccagnini (2008) citado por Morillo \& Quijano (2016) define que las prácticas pedagógicas son:

aquellas productoras de sujetos a partir de otros sujetos, es decir, se trata de una mediación, el rol de un sujeto mediador (sujeto pedagógico), que se relaciona con otro sujeto (educando) de esta relación surgen situaciones educativas complejas las que encuadran y precisan una pedagogía. El sujeto pedagógico se entiende como un ser histórico definido por la institución escolar, capaz de acercarse a esa complejidad de un universo sociocultural con una perspectiva más amplia que lo define con un sin número de articulaciones posibles entre educador, educando, saberes y la configuración de los espacios educativos que legitiman su propia pedagogía. (pág. 323).

Se evidencia que la elaboración e implementación de los proyectos pedagógicos de aula, aportaron al crecimiento personal y profesional desde el reconocimiento del saber ser, saber hacer y saber de los educadores, no obstante, es importante resaltar que el programa de pedagogía para profesionales no licenciados de UNIMINUTO, brindó una formación teóricopráctico esencial para que estos profesionales adquieran las herramientas necesarias para 
fortalecer sus habilidades, destrezas y brindar una formación de calidad a sus estudiantes, teniendo en cuenta sus necesidades e intereses que trascienden su experiencia educativa. Además, el PPA permite proporcionar herramientas, prácticas, teóricas y metodológicas para que los profesionales desarrollen competencias de liderazgo, trabajo en equipo, comunicación asertiva, solución pacífica de conflictos escolares e innoven sus métodos y estrategia de enseñanza- aprendizaje (González E. et al. 2020).

Por lo anterior, es pertinente continuar con la herramienta de elaboración e implementación de los proyectos pedagógicos de aula, debido a que le permite al PNL reflexionar sobre sus acciones, asumir el rol de mediador de aprendizaje y reconocerse como un ser en constante mejora de sus prácticas.

Adicional a lo anterior Barón, Cancino, Guativa \& García (2011) destacan que cuando se trabaja con seres humanos, se vive más en las incertidumbres que en las certidumbres, cualquier cosa puede pasar, la planeación de las clases es una ruta orientadora, pero ella se modifica estructuralmente por las situaciones que acontecen en el aula, por ejemplo, tener una confrontación con algún estudiante en clase, puede generar experiencias que condicionan la manera de ver la vida. Cuando los profesionales no licenciados reflexionan sobre estas situaciones, reconocen que en ellos también hay un llamado a transformar vidas. Ver gráfico (\# 6).

De igual forma, Rodríguez (2017) sostiene en su investigación que el 70\% de los estudiantes está de acuerdo, que la formación en pedagogía le permitió modificar la concepción inicial que tenía en educación, el 82\% de los estudiantes percibió la profesión docente como una mejor oportunidad laboral que su formación inicial, el $63 \%$ de los estudiantes afirma que los resultados de los procesos formativos en el programa cumplieron con sus expectativas. En esta misma línea se encuentra la investigación de Cuervo (2013), donde se explora los aciertos y falencias del quehacer del profesional docente, así mismo, se analizan los procesos de formación inicial de los docentes (saber pedagógico y las competencias básicas).

Los autores mencionados concuerdan que la importancia de la formación de los educadores debe estar dirigida hacia la responsabilidad de la formación docente, esta no solo recae en la política educativa, sino que las universidades con programas de formación docente están llamadas a contribuir en la calidad de los programas que conducen a obtener 
un título o reconocimiento que les acredite como idóneos para ejercer la docencia si no que se les permita al PNL visibilizar un reto educativo que le permita una reflexión constante que enriquezca su proceso no solo profesional si no personal.

\section{Conclusiones}

El Programa de Pedagogía para Profesionales No Licenciados de la Corporación Universitaria Minuto de Dios- UNIMINUTO, contribuyó en las transformaciones pedagógicas de los Profesionales No Licenciados, siendo estas positivas en la construcción de la identidad de maestras/os, con el propósito de mejorar la calidad educativa y formar seres humanos íntegros que tanto reclaman las diversas sociedades en la actualidad.

Los contenidos curriculares y prácticas pedagógicas de los seminarios desarrollados en el programa permitieron a los Profesionales No Licenciados adquirir herramientas suficientes para trasformar sus aulas de clases en escenarios creativos, autónomos y didácticos, en los que primen los interaprendizajes.

Se evidencia que los Profesionales No Licenciados ya no se auto observan como la única autoridad en el aula de clases, sino que se conciben como ese facilitador de oportunidades que involucra diferentes herramientas ya sean tecnológicas o teóricas para fortalecerlas competencias de sus prácticas pedagógicas en comunicación con sus estudiantes.

Los proyectos pedagógicos de aula permitieron al Profesional No Licenciados establecer métodos, técnicas de enseñanza y actividades que generan interacciones entre necesidades e intereses, favoreciendo el contexto educativo.

Con la elaboración de los proyectos, se identificaron falencias y fortalezas del grupo de trabajo, permitiendo entender la manera de abordar, socializar y planear las actividades pedagógicas para comunicar el tipo de conocimiento que los estudiantes realmente desean aprender. Además, el tipo de acercamiento entre los actores de los procesos educativos, se caracterizaron por la dinámica, flexibilidad, adaptación y orientación, dando horizontes que trasciendan el cumplimiento de los objetivos.

En los hallazgos se reconoce que cada participante es un mundo diferente, cada uno se expresó de acuerdo con sus vivencias, sentimientos y emociones; los profesionales reconocieron que la experiencia es significativa, que la dimensión formativa en el contexto 
académico es fundamental, pero no la única, razón por la cual, es crucial el reconocimiento incondicional del otro como legítimo y válido en los procesos de aprendizajes.

Para terminar, es importante destacar que múltiples investigaciones en el contexto de la formación de Profesionales No Licenciados develan la necesidad de crear estrategias comunicativas que optimicen los diálogos epistémicos entre los Profesionales No Licenciados, docentes y directivos delos programas de pedagogía para profesionales no licenciados, para dar continuidad a la construcción e implementación de comunidades de aprendizajes, como la propuesta en la tesis “Transformaciones de las Prácticas Pedagógicas de los graduados del Programa de Pedagogía para Profesionales No Licenciados" permitiendo el fortalecimiento tanto de los programas como de las políticas públicas en todo el país.

\section{Referencias}

Aktuna, H.E.; Oliveras Contreras, M.L. (2016). La enseñanza basada en antecedentes socioculturales de los alumnos: un método de eliminación de la desigualdad para lograr el aprendizaje significativo. ReiDoCrea, 5: 130-145 10.30827/Digibug.41788

Artigas, W., \& Robles, M. (2010). Metodología de la investigación: Una discusión necesaria en Universidades Zulianas. Revista Digital Universitaria, 11, 1-17. http://www.revista.unam.mx/vol.11/num11/art107/art107.pdf

Assmann, H. (2002) Placer y ternura en la educación. Hacia una sociedad aprendiente. Madrid: Narcea.

Barón, B. (2017). Conversaciones (prácticas vitales en educación superior). Bogotá: Aula Humanidades.

Barón, B., Cancino, J., García D y Guativa J. (2011). La comunicación verbal en la escuela: más allá de las palabras. Una muestra de la actividad realizada por maestros formados en investigación. Actualidades Pedagógicas No. 57. Ediciones Unisalle. DOI: https://doi.org/10.19052/ap.555

Bonilla, E., \& Rodríguez, P. (2005). Más allá del dilema de los métodos: la investigación en ciencias sociales (Tercera edición ampliada y revisada ed.) [Libro electrónico]. Norma. $\quad$ https://laboratoriociudadut.files.wordpress.com/2018/05/mas-alla-deldilema-de-los-metodos.pdf 
Borjas, J. E. (2020). Validez y confiabilidad en la recolección y análisis de datos bajo un enfoque cualitativo. Trascender, Contabilidad $Y$ Gestión, 15, 79-97. https://doi.org/10.36791/tcg.v0i15.90

Bracho Pérez, K. J. (2019). 8. Competencias investigativas del docente para el fortalecimiento de su praxis pedagógica. Revista EDUCARE - UPEL-IPB - Segunda Nueva Etapa 2.0, 23(1), 188-201. https://doi.org/10.46498/reduipb.v23i1.16

Buendia, L., Bravo, C., Coaut, M., Hernández, P., \& Fuensanta, C. (1998). Métodos de Investigación en Psicopedagogía. [Libro electrónico]. McGraw-Hill Education. http://www.sidalc.net/cgi-

bin/wxis.exe/?IsisScript=AGRIUAN.xis\&method=post $\&$ formato=2\&cantidad=1\&e xpresion $=\mathrm{mfn}=027450$

Caldeiro-Pedreira, M.-C., \& Castro-Zubizarreta, A. (2020). ¿Cómo enfrentar la educación en la era de la interactividad? Praxis Pedagógica, 20(26), 33-53. https://doi.org/10.26620/uniminuto.praxis.20.26.2020.33-53

Di Franco, N. B., Gentile, C., \& Di Franco, M. G. (2013). Las prácticas profesionales docentes en el profesorado de matemática: entre el acompañamiento académico y las tutorías profesionales. Actualidades Pedagógicas, 61, 139-153. https://doi.org/10.19052/ap.2335.

Durán, F. Á., Acosta, D. G., \& Espinel, Ó. A. (2014). Experiencia docente de profesionales no licenciados en la escuela pública del Distrito. Actualidades Pedagógicas,63, 3960.https://doi.org/10.19052/ap.2722.

Figueroa, A. (2018). Innovaciones en la formación inicial docente y los desafíos para el desarrollo profesional docente. Revista Saberes Educativos, 2, 103-119. https://doi.org/10.5354/2452-5014.2019.52120

García, C. (Ed.). (2019). Reflexiones pedagógicas de los PNL en su ejercicio profesional en la educación en la ciudad de Bogotá (N.o 66). Universidad Pedagógica Nacional. http://repository.pedagogica.edu.co/bitstream/handle/20.500.12209/11376/TO23654.pdf? sequence $=1 \&$ is Allowed $=\mathrm{y}$

Hernández, S. (2014). Metodología de la investigación - Sexta Edición. En C. Fernández \& L. Baptista (Eds.), Metodología de la investigación - Sexta Edición (Sexta ed., pp. 170-191). McGraw-Hill Education. 
Jarpa, M., Haas, V., \& Collao, D. (2017). Escritura para la reflexión pedagógica: rol y función del Diario del Profesor en Formación en las Prácticas Iniciales. Estudios pedagógicos (Valdivia), 43(2), 163-178.https://doi.org/10.4067/s0718-07052017000200009.

Jarpa, M. (2019). Escritura académica para el desarrollo de la reflexión pedagógica en la formación docente: la Crónica Docente-Directiva. Íkala, 24(1), 85-101. https://doi.org/10.17533/udea.ikala.v24n01a04

Juliao, C. G., \& Barón-Velandia, B. (2013). El enfoque praxeológico. Praxis Pedagógica, 13(14),

$141-145$. https://doi.org/10.26620/uniminuto.praxis.13.14.2013.141-145

León, A., Risco, E. y Alarcón, C. (2018). Aprendizajes que promueven los profesores en un enfoque curricular por competencia en educación superior. Praxis Pedagógica, 8(23), 1-21 https://doi.org/10.26620/uniminuto.praxis.18.23.2018.108-126

López, L. (2013). La hermenéutica y sus implicaciones en el proceso educativo / The implications of hermeneutics in the educational process. Sophía, 1(15), 86. https://doi.org/10.17163/soph.n15.2013.02

MEN. (2002, 19 junio). Decreto 1278 de junio 19 de 2002 - Ministerio de Educación Nacional de Colombia. Mineducaciòn. https://www.mineducacion.gov.co/1759/w3article-86102.html?_noredirect=1.

Morillo, O. A., \& Quijano, Ó. (2016). Concepciones y prácticas docentes sobre la diversidad como base para la innovación pedagógica. Un estudio de caso en el colegio Vasco Núñez de Balboa. Plumilla Educativa, 18(2), 318-336. https://doi.org/10.30554/plumillaedu.18.1971.2016.

Olmos, A., Sánchez, M., \& Correa, A. S. (2016). Reflexión y transformación pedagógica. Reconocimiento a la otredad. Revista Iberoamericana de Educación, 71(1), 928.https://doi.org/10.35362/rie71145

Paredes, Z., \& Velázquez, D. (2020). Nuevo papel de la didáctica implementada en las aulas universitarias. Ciencia Latina Revista Científica Multidisciplinar, 4(2), 1111-1122. https://doi.org/10.37811/cl_rcm.v4i2.142

Pérez, L., \& Ochoa, A. (2017). Participación de los estudiantes en una escuela secundaria. Revista Mexicana de Investigación Educativa,22, 179-207. http://www.scielo.org.mx/pdf/rmie/v22n72/1405-6666-rmie-22-72-00179.pdf 
Pérez-Rodríguez, N. (2019). Programas de Formación Docente en Educación Superior en el contexto español. Revista Investigación en la Escuela, 97, 1-17. https://doi.org/10.12795/ie.2019.i97.01

Pineda, E., \& Orozco, P. (2016). El currículo praxeológico como interés de conocimiento $\begin{array}{llll}\text { emancipatorio. } & \text { Praxis } & \text { Pedagógica, } & 16(18),\end{array}$ http://dx.doi.org/10.26620/uniminuto.praxis.16.18.2016.11-25

Quintana, A., \& Montgomery, W. (2006). Metodología de Investigación Científica Cualitativa. Tópicos de 47-84. http://www.ubiobio.cl/miweb/webfile/media/267/3634305-Metodologia-deInvestigacion-Cualitativa-A-Quintana.pdf

Rodríguez, H. M. (2006). Práctica pedagógica. Una tensión entre la teoría y la práctica. Pedagogía y Saberes, 24(24), 19-25. https://doi.org/10.17227/01212494.24pys19.25 Rodríguez-Pérez, M. V., \& Hinojo-Lucena, F. J. (2017). Incidencia de los Programas de Formación Pedagógica en el Perfil de los Profesionales No Licenciados en la Facultad de Educación de UNIMINUTO. Formación universitaria, 10(5), 17-28. https://doi.org/10.4067/s0718-50062017000500003.

Rodríguez-Pérez, M. V., González-Riveros, A. M., Ibarra-Cortés, E. F., \& Páez-Angarita, A. (2018). Movilidad Académica en Programas de Formación de Educadores Infantiles de Universidades en Bogotá, Colombia. Formación universitaria, 11(4), 53-64. https://dx.doi.org/10.4067/S0718-50062018000400053

Rodríguez-Pérez, M. V., \& Hinojo-Lucena, F. J. (2017a). Incidencia de los Programas de Formación Pedagógica en el Perfil de los Profesionales No Licenciados en la Facultad de Educación de UNIMINUTO. Formación universitaria, 10(5), 17-28. https://doi.org/10.4067/s0718-50062017000500003

Ropa, B. (2014). Administración de la calidad en los servicios educativos. Horizonte de la Ciencia, 4(6), 67-73.https://doi.org/10.26490/uncp.horizonteciencia.2014.6.93

Ryen, 2013; \& Grinnell y Unrau, (2011). La entrevista en profundidad. Recuperado de http://www.redalyc.org/pdf/351/35124304004.pdf

Torres, J., \& Flores Ferrer, K. M. (2018). 6. Entorno Virtual de Aprendizaje (EVA) para la administración b-learning del curso Tecnología y Práctica de Mercadeo I. Revista EDUCARE - UPEL-IPB - Segunda Nueva Etapa 2.0, 21(2), 68-78. 
https://doi.org/10.46498/reduipb.v21i2.54Tamayo, A. (2004). La formación pedagógica del docente universitario. Pedagogía y Saberes, 21, 2935.https://doi.org/10.17227/01212494.21pys29.35

Tolozano, S. E., Lara, L. M., \& Illescas, S. A. (2015). Formación pedagógica del profesorado de las carreras tecnológicas del Instituto Bolivariano de Tecnología de $\begin{array}{llll}\text { Ecuador. Ciencia } & y & \text { Sociedad, } 40(1), & 109-132 .\end{array}$ https://doi.org/10.22206/cys.2015.v40i1.pp109-132

UNESCO. (2005, febrero). Educación para Todos: El imperativo de la calidad ( $\left.\mathrm{N}^{\mathrm{0}} 3\right)$. https://web.oas.org/childhood/ES/Lists/Recursos\%20\%20Bibliografia/Attachments/ 91/102.pd

Varela-Ordorica, S. A., \& Valenzuela-González, J. R. (2020). Uso de las tecnologías de la información y la comunicación como competencia transversal en la formación inicial de docentes. Revista Electrónica $\quad$ Educare, 24(1), 1-20. https://doi.org/10.15359/ree.24-1.10

Varón, V., Martínez, S., León, Y. P., \& Barón-Velandia, B. (2020). Pedagogía de la alteridad: entre la educación como práctica social y el ejercicio intelectual. Revista Insignare Scientia - RIS, 3(2), 484-508. https://doi.org/10.36661/2595-4520.2020v3i2.11582

Zaccagnini, M. (2008). Impacto de los paradigmas pedagógicos históricos en las prácticas educativas contemporáneas. Revista Iberoamérica de educación ISSN: 1681-5653. http://www.rieoei.org/deloslectores/443Zaccagnini.pdf.

Zuluaga, O. L. (2005). Foucault, La Pedagogía y La Educación: Pensar de Otro Modo (Spanish Edition) (Primera Edición). Delfín Ltda.

Zurita Aguilera, M. S. (2020). El aprendizaje cooperativo y el desarrollo de las habilidades cognitivas. Revista EDUCARE - UPEL-IPB - Segunda Nueva Etapa 2.0, 24(1), 5174. https://doi.org/10.46498/reduipb.v24i1.1226 